\title{
ABSOLUTE CONTINUITY OF THE SCHRÖDINGER OPERATOR SPECTRUM IN A MULTIDIMENSIONAL CYLINDER
}

\author{
I. KACHKOVSKII AND N. FILONOV
}

\begin{abstract}
The Schrödinger operator $-\Delta+V$ in a $d$-dimensional cylinder, $d \geq 3$, is considered with various boundary conditions. Under the assumption that the potential $V$ is periodic with respect to the "longitudinal" variables and $V \in L_{d-1, \text { loc }}$, it is proved that the spectrum of the Schrödinger operator is absolutely continuous.
\end{abstract}

\section{$\S 1$. INTRODUCTION}

Let $U \subset \mathbb{R}^{k}$ be a bounded domain, and let

$$
\Xi=U \times \mathbb{R}^{m} \subset \mathbb{R}^{d}, \quad d=k+m, \quad m \geq 1 .
$$

Our purpose in the present paper is to prove the absolute continuity of the spectrum of the Schrödinger operator $H=-\Delta+V$ in the cylinder $\Xi$ (see Theorem 3.3 and Corollaries 3.4, 3.5 below). The points of $\Xi$ are denoted by $(x, y), x \in U, y \in \mathbb{R}^{m}$. Let $\Gamma$ be a lattice in $\mathbb{R}^{m}$,

$$
\Gamma=\left\{l=\sum_{j=1}^{m} l_{j} b_{j}, \quad l_{j} \in \mathbb{Z}\right\},
$$

where $\left\{b_{j}\right\}_{j=1}^{m}$ is a fixed (not necessarily orthogonal) basis in $\mathbb{R}^{m}$. It is assumed that the potential $V$ is a real-valued function periodic with respect to the "longitudinal" variables,

$$
V(x, y+l)=V(x, y), \quad l \in \Gamma, \quad(x, y) \in \Xi .
$$

On the boundary of $\Xi$ we impose either the Dirichlet condition $\left.u(x, y)\right|_{\partial \Xi}=0$ or the third type boundary condition

$$
\left.\left(\frac{\partial u(x, y)}{\partial \nu}+\sigma(x) u(x, y)\right)\right|_{\partial \Xi}=0
$$

where $\partial / \partial \nu$ is the normal derivative, and $\sigma$ is a real-valued function on $\partial U$. We only consider the case where $\sigma$ does not depend on $y$ (see also Subsection 7.3 below). The case of $\sigma=0$ gives us the Neumann boundary condition.

We list some of the previously known results concerning the absolute continuity of the spectrum of $H$. Our review does not claim to be exhaustive. Usually, sufficient conditions for $H$ to be absolutely continuous are formulated in terms of $V$ belonging either to the

2000 Mathematics Subject Classification. Primary 35P05.

Key words and phrases. Absolute continuity of the spectrum, Schrödinger operator, periodic coefficients.

The research of the first author was supported by EPSRC (grant GR/T25552/01) and by RFBR (grant no. 08-01-00209-a). 
function space $L_{p}(U \times \Omega)$ or to $L_{p, \infty}^{0}(U \times \Omega)$. Here

$$
\Omega=\left\{y=\sum_{j=1}^{m} y_{j} b_{j}, \quad y_{j} \in(0,1)\right\}
$$

is an elementary cell of $\Gamma$, and $L_{p, \infty}^{0}$ is the weak Lorentz class. We recall that if $M$ is a set of finite measure, then we have the following embeddings: $L_{p}(M) \subset L_{p, \infty}^{0}(M) \subset$ $L_{p-\varepsilon}(M), \varepsilon>0$.

The case of the entire space $\left(k=0, m=d, \Xi=\mathbb{R}^{d}\right)$ is well developed (see, for example, [13, 6, 2, 15]). If

$$
V \in L_{p}(\Omega), p>1 \text { for } d=2, \quad V \in L_{d / 2, \infty}^{0}(\Omega) \text { for } d \geq 3,
$$

then the spectrum of $H$ is absolutely continuous.

The case of $k=1$ ( $U$ is a line segment, $\Xi$ is a plane parallel layer) was considered in 9]: for $d=2,3,4$, the same conditions are sufficient, and if $d \geq 5$, then the condition is $V \in L_{d-2, \infty}^{0}(U \times \Omega)$. The case of $\sigma$ periodically dependent on $y$ was also treated in that paper. The corresponding sufficient conditions are

$$
\begin{gathered}
\sigma \in L_{p}(\partial U \times \Omega), p>1 \text { for } d=2, \quad \sigma \in L_{2, \infty}^{0}(\partial U \times \Omega) \text { for } d=3, \\
\sigma \in L_{2 d-2}(\partial U \times \Omega) \text { for } d \geq 4 .
\end{gathered}
$$

In the case of $k \geq 2$, the absolute continuity of the spectrum of $H$ was proved in the book [4] (Theorem 5.4.9 therein) for $V \in L_{\infty}(\Xi), \partial U \in C^{\infty}$; for the third type boundary condition, $\sigma$ only depends on $x$. Also, in the unpublished paper 3 , the problem was solved in the case of Dirichlet and Neumann boundary conditions for $m=1, \partial U \in C^{2}$, and $V \in L_{2(d-2), \infty}^{0}(U \times \Omega)$.

We consider the general case ( $m$ is an arbitrary natural number, no restrictions are imposed on the boundary $\partial U$ in the Dirichlet case, and the boundary is Lipschitz in the case of the third type boundary condition) and prove that if $V \in L_{d-1}(U \times \Omega)$ and $d \geq 3$, then the spectrum of $H$ is absolutely continuous. More precisely, our result is a little more general. Namely, we consider $H$ of the form $H=-\Delta_{y}+\mathcal{A}+V$, where $\mathcal{A}$ is a selfadjoint semibounded operator in $L_{2}(U)$ with discrete spectrum. The spectrum of such an $H$ is absolutely continuous (see Theorem 3.3 for a precise formulation). The result mentioned above for the "usual" Schrödinger operator is obtained as a corollary if we take $\mathcal{A}$ to be the Laplace operator in $U$ with the corresponding boundary conditions (see Corollaries 3.4 and 3.5).

The eigenvalues and eigenfunctions of the nonperturbed Laplace operator $-\Delta$ in $U \times \Omega$ with various boundary conditions are very important for proving the absolute continuity of the spectrum. If $k=0$ or $k=1$, then the set $U \times \Omega$ is a parallelepiped. The corresponding eigenfunctions $\left\{\varphi_{j}\right\}_{j=1}^{\infty}$ are linear combinations of the imaginary exponents, so that they are uniformly bounded. In accordance with the interpolation theory, this implies that the corresponding discrete Fourier transform is bounded as an operator acting from $l_{p}$ to $L_{p^{\prime}}$. This fact was used in the course of the proofs of all above-mentioned results. However, for $k \geq 2$, the norms $\left\|\varphi_{j}\right\|_{L_{\infty}}$ are unbounded in general. In the present paper we use embedding theorems for anisotropic Sobolev spaces instead of interpolation theory (see the proof of Theorem 4.5 below).

The methods we use do not allow us to consider the case of the magnetic Schrödinger operator or the case of a variable metric. The corresponding results for the case of the entire space $\left(\Xi=\mathbb{R}^{d}\right)$ and for the case of a two-dimensional waveguide $(k=m=1)$ can be found in [2, 11, 17; see also the references therein.

We use the following notation: $\operatorname{spec}(A)$ is the spectrum of an operator $A ;\langle\cdot, \cdot\rangle$ and $(\cdot, \cdot)$ are the standard scalar products in $\mathbb{C}^{m}$ and in $L_{2}$, respectively; $W_{p}^{1}, H^{1}=W_{2}^{1}, H_{0}^{1}$ 
are Sobolev spaces; $\widetilde{H}^{1}(\Omega)$ is the subspace of $H^{1}(\Omega)$ formed by the functions satisfying the periodic boundary condition.

The authors are grateful to Prof. T. A. Suslina for drawing their attention to the question addressed in the present paper and to Prof. A. B. Pushnitskiı for useful discussions.

\section{$\S 2$. Preliminaries}

2.1. Free operator. Let $U \subset \mathbb{R}^{k}$ be an open bounded subset. Assume that $a$ is a closed, semibounded, and densely defined quadratic form in the Hilbert space $L_{2}(U)$. Let its domain be $D[a]$, and let $\mathcal{A}$ be the corresponding selfadjoint operator. We denote by $\mu_{1}$ the exact lower bound of $a$,

$$
\mu_{1}=\inf _{f \neq 0} \frac{a[f]}{\|f\|_{L_{2}}^{2}}=\min \operatorname{spec}(\mathcal{A}) .
$$

Fix any $\gamma>-\mu_{1}$. The space $D[a]$ with the norm

$$
\|f\|_{D[a]}=\sqrt{a[f]+\gamma\|f\|_{L_{2}}^{2}}
$$

is a Hilbert space. We assume that the functions in $D[a]$ admit an extension to functions of class $H^{1}\left(\mathbb{R}^{k}\right)$ and, in particular, $D[a] \subset H^{1}(U)$. More precisely, we impose the following condition on $D[a]$.

Condition 2.1. There exists a linear extension operator

$$
\Pi_{0}: L_{2}(U) \rightarrow L_{2}\left(\mathbb{R}^{k}\right),\left.\quad\left(\Pi_{0} f\right)\right|_{U}=f,
$$

such that if $f \in D[a]$, then $\Pi_{0} f \in H^{1}\left(\mathbb{R}^{k}\right)$ and

$$
\left\|\Pi_{0} f\right\|_{H^{1}\left(\mathbb{R}^{k}\right)}^{2} \leq C_{0}\left(a[f]+\gamma\|f\|_{L_{2}(U)}^{2}\right) .
$$

With this condition, the embedding $D[a] \subset L_{2}(U)$ is compact, and the spectrum of $\mathcal{A}$ is discrete.

Put $\Xi=U \times \mathbb{R}^{m}$. We consider the Hilbert space $L_{2}(\Xi)=L_{2}\left(\mathbb{R}^{m} ; L_{2}(U)\right)$ and the quadratic form

$$
h_{0}[u]=\int_{\mathbb{R}^{m}}\left(\left\|\nabla_{y} u(\cdot, y)\right\|_{L_{2}(U)}^{2}+a[u(\cdot, y)]\right) d y
$$

on the domain

$$
D\left[h_{0}\right]=H^{1}\left(\mathbb{R}^{m} ; L_{2}(U)\right) \cap L_{2}\left(\mathbb{R}^{m} ; D[a]\right) .
$$

Example 2.2. Let $D[a]=H^{1}(U)$. Suppose the boundary $\partial U$ is Lipschitz. Then Condition 2.1 is fulfilled (see, e.g., [8]), and $D\left[h_{0}\right]=H^{1}(\Xi)$.

Example 2.3. Let $D[a]=H_{0}^{1}(U)$, where $U \subset \mathbb{R}^{n}$ is a bounded open subset. Condition 2.1 is fulfilled again (it suffices to consider the zero extension), and $D\left[h_{0}\right]=H_{0}^{1}(\Xi)$.

The nonperturbed Schrödinger operator in the cylinder $\Xi$ is a selfadjoint operator $H_{0}$ that corresponds to the quadratic form (2.2), (2.3). Formally, we may write

$$
H_{0}=-\Delta_{y}+\mathcal{A} \text {. }
$$

The spectrum of $H_{0}$ can be described easily. Let $\left\{\psi_{j}\right\}_{j=1}^{\infty}$ be the basis of eigenfunctions of $\mathcal{A}$ in $L_{2}(U)$, and let $\left\{\mu_{j}\right\}_{j=1}^{\infty}$ be the corresponding eigenvalues. The space $L_{2}(\Xi)$ can be decomposed into a direct sum

$$
L_{2}(\Xi)=\bigoplus_{j=1}^{\infty}\left\{u(x, y)=\psi_{j}(x) f(y), f \in L_{2}\left(\mathbb{R}^{m}\right)\right\} .
$$


This decomposition reduces $H_{0}$. In each of the summands of the right-hand side, the operator acts as a Laplace operator in $\mathbb{R}^{m}$ "shifted" by $\mu_{j}$. So, $\operatorname{spec}\left(H_{0}\right)=\left[\mu_{1}, \infty\right)$ and the spectrum is absolutely continuous.

2.2. The extension operator. Assume that an open subset $U \subset \mathbb{R}^{k}$ and a quadratic form $a$ acting in $L_{2}(U)$ satisfy Condition 2.1. Let $\left\{b_{j}\right\}_{j=1}^{m}$ be a basis in $\mathbb{R}^{m}$, and let $\Omega \subset \mathbb{R}^{m}$ be the cell (1.4). Consider a function $u(x, y)$ on $U \times \Omega$. For any fixed $y \in \Omega$, the operator $\Pi_{0}$ occurring in Condition 2.1 extends $u(\cdot, y)$ from $U$ to $\mathbb{R}^{k}$. The new function on $\mathbb{R}^{k} \times \Omega$ constructed this way is denoted by $\Pi u$. We denote by $\partial_{j}$ the derivative in the direction $b_{j}$.

Lemma 2.4. The extension operator $\Pi: L_{2}(U \times \Omega) \rightarrow L_{2}\left(\mathbb{R}^{k} \times \Omega\right)$ commutes with the derivations with respect to the variables $y$ : if $u \in L_{2}(U \times \Omega)$ and $\partial_{j} u \in L_{2}(U \times \Omega)$, then $\partial_{j}(\Pi u)$ exists and

$$
\partial_{j}(\Pi u)=\Pi\left(\partial_{j} u\right) .
$$

Proof. It is easily seen that (2.4) holds true if $u \in C^{1}\left(\bar{\Omega} ; L_{2}(U)\right)$. Furthermore, for any function $u \in L_{2}\left(\Omega ; L_{2}(U)\right)$ with $\partial_{j} u \in L_{2}\left(\Omega ; L_{2}(U)\right)$, there exists a sequence of $v_{k} \in C^{1}\left(\bar{\Omega} ; L_{2}(U)\right)$ such that

$$
v_{k} \rightarrow u, \quad \partial_{j} v_{k} \rightarrow \partial_{j} u \quad \text { in } \quad L_{2}\left(\Omega ; L_{2}(U)\right), \quad k \rightarrow \infty .
$$

Then the statement of the lemma follows from the fact that the generalized derivation operator is closed.

Lemma 2.5. Let $\Pi$ be the extension operator described above, and let

$$
u \in H^{1}\left(\Omega ; L_{2}(U)\right) \cap L_{2}(\Omega ; D[a]) .
$$

Then $\Pi u \in H^{1}\left(\mathbb{R}^{k} \times \Omega\right)$ and

$$
\|\Pi u\|_{H^{1}\left(\mathbb{R}^{k} \times \Omega\right)}^{2} \leq C_{1} \int_{\Omega}\left(\left\|\nabla_{y} u(\cdot, y)\right\|_{L_{2}(U)}^{2}+a[u(\cdot, y)]+\gamma\|u(\cdot, y)\|_{L_{2}(U)}^{2}\right) d y,
$$

where $\gamma$ is as in (2.1), $C_{1}=\max \left(C_{0},\left\|\Pi_{0}\right\|_{L_{2} \rightarrow L_{2}}^{2}\right)$, and $C_{0}$ is the constant from Condition 2.1.

Proof. This follows from the preceding lemma and the properties of $\Pi_{0}$ :

$$
\begin{aligned}
& \|\Pi u\|_{H^{1}\left(\mathbb{R}^{k} \times \Omega\right)}^{2}=\int_{\Omega}\left(\left\|\Pi_{0} u(\cdot, y)\right\|_{H^{1}\left(\mathbb{R}^{k}\right)}^{2}+\left\|\nabla_{y} \Pi u(\cdot, y)\right\|_{L_{2}\left(\mathbb{R}^{k}\right)}^{2}\right) d y \\
& \quad \leq \int_{\Omega}\left(C_{0}\left(a[u(\cdot, y)]+\gamma\|u(\cdot, y)\|_{L_{2}(U)}^{2}\right)+\left\|\Pi_{0}\right\|_{L_{2} \rightarrow L_{2}}^{2}\left\|\nabla_{y} u(\cdot, y)\right\|_{L_{2}(U)}^{2}\right) d y .
\end{aligned}
$$

Lemma 2.5 and the embedding theorem, $H^{1}\left(\mathbb{R}^{k} \times \Omega\right) \subset L_{\frac{2 d}{d-2}}\left(\mathbb{R}^{k} \times \Omega\right)$, imply the following statement.

Corollary 2.6. Suppose $d \geq 3$ and $u \in H^{1}\left(\Omega ; L_{2}(U)\right) \cap L_{2}(\Omega ; D[a])$. Then

$$
\|u\|_{L_{\frac{2 d}{d-2}}^{2}(U \times \Omega)}^{2} \leq C \int_{\Omega}\left(\left\|\nabla_{y} u(\cdot, y)\right\|_{L_{2}(U)}^{2}+a[u(\cdot, y)]+\gamma\|u(\cdot, y)\|_{L_{2}(U)}^{2}\right) d y .
$$




\section{§3. Formulation OF THE RESUlT}

3.1. General case. Everywhere in the sequel we assume that $d=k+m \geq 3$ (above it was mentioned that the case where $d=2$ is well developed). We need the following fact to show that the Schrödinger operator $H=H_{0}+V$ with a periodic potential $V \in L_{d / 2, \text { loc }}(\Xi)$ is well defined.

Lemma 3.1. Let $(M, d \mu)$ be a measurable space with $\sigma$-finite measure, and let $V \in$ $L_{p}(M), 1 \leq p<\infty$. Then for any $\delta>0$ there exists a constant $c(\delta)$ such that

$$
\int_{M}|V f g| d \mu \leq \delta\|f\|_{L_{2 p^{\prime}}(M)}\|g\|_{L_{2 p^{\prime}}(M)}+c(\delta)\|f\|_{L_{2}(M)}\|g\|_{L_{2}(M)}, \quad f, g \in L_{2 p^{\prime}}(M),
$$

where $p^{\prime}$ is the index conjugate to $p$.

Proof. The function $V$ can be expressed in the form

$$
V=V_{1}+V_{2}, \quad\left\|V_{1}\right\|_{L_{p}(M)} \leq \delta, \quad V_{2} \in L_{\infty}(M) .
$$

Hölder's inequality yields

$$
\int_{M}|V f g| d \mu \leq \delta\|f\|_{L_{2 p^{\prime}}(M)}\|g\|_{L_{2 p^{\prime}}(M)}+\left\|V_{2}\right\|_{L_{\infty}(M)}\|f\|_{L_{2}(M)}\|g\|_{L_{2}(M)} .
$$

Lemma 3.2. Let $\Gamma$ be the lattice (1.1) in $\mathbb{R}^{m}$, let $\Omega$ be the elementary cell (1.4), and let $V$ be a real-valued function on $\Xi=U \times \mathbb{R}^{m}$ satisfying the periodicity condition (1.2) and such that $V \in L_{d / 2}(U \times \Omega)$. Then for any $\varepsilon>0$ there exists a constant $c(\varepsilon)$ such that

$$
\begin{array}{lr}
\int_{U \times \Omega}\left|V\left\|\left.u\right|^{2} d x d y \leq \varepsilon \int_{\Omega}\left(\left\|\nabla_{y} u(\cdot, y)\right\|_{L_{2}(U)}^{2}+a[u(\cdot, y)]\right) d y+c(\varepsilon)\right\| u \|_{L_{2}(U \times \Omega)}^{2},\right. & u \in D\left[h_{0}\right], \\
\int_{\Xi}\left|V\left\|\left.u\right|^{2} d x d y \leq \varepsilon h_{0}[u]+c(\varepsilon)\right\| u \|_{L_{2}(\Xi)}^{2}, \quad u \in D\left[h_{0}\right],\right. &
\end{array}
$$

where $h_{0}$ is the form introduced in (2.2), (2.3).

Proof. Estimate (3.1) follows from the preceding lemma with $p=d / 2$ and Corollary 2.6. Summing (3.1) over all the cells, we get (3.2).

Consider a quadratic form

$$
h[u]=h_{0}[u]+\int_{\Xi} V|u|^{2} d x d y, \quad D[h]=D\left[h_{0}\right]
$$

in $L_{2}(\Xi)$. Lemma 3.2 shows that the form $h$ is closed and semibounded. By definition, the Schrödinger operator in the cylinder $\Xi$ with an electric potential $V$ is the selfadjoint operator $H=H_{0}+V$ corresponding to $h$. Now we can formulate our main result.

Theorem 3.3. Suppose $U \subset \mathbb{R}^{k}$ is a bounded open subset, $\Xi=U \times \mathbb{R}^{m}$, and $d=$ $k+m \geq 3$. Let $\Gamma$ be the lattice (1.1) in $\mathbb{R}^{m}$, and let $\Omega$ be its elementary cell (1.4). Let $a$ be a closed semibounded quadratic form in $L_{2}(U)$ satisfying Condition 2.1, and let $h_{0}$ be a quadratic form in $L_{2}(\Xi)$ defined by (2.2), (2.3). Suppose the real-valued potential $V$ satisfies the periodicity condition (1.2) and $V \in L_{d-1}(U \times \Omega)$. Then the spectrum of the operator $H$ corresponding to the quadratic form (3.3) is absolutely continuous.

We note that the conditions on the potential $V$ can be relaxed if we formulate them in terms of anisotropic function spaces; see Subsection 7.1 below. 
3.2. The "usual" Schrödinger operator. Now we reformulate our main result for the operator $-\Delta+V$, i.e., for the case where the operator $\mathcal{A}$ in $L_{2}(U)$ is the $k$-dimensional Laplace operator with various boundary conditions.

Corollary 3.4. Let $\Xi, \Gamma$, and $V$ satisfy the conditions of Theorem 3.3, Then the spectrum of the Schrödinger operator $H_{D}$ corresponding to the quadratic form

$$
h_{D}[u]=\int_{\Xi}\left(|\nabla u(x, y)|^{2}+V(x, y)|u(x, y)|^{2}\right) d x d y, \quad D\left[h_{D}\right]=H_{0}^{1}(\Xi),
$$

is absolutely continuous.

Indeed, in this case,

$$
a[f]=\int_{U}|\nabla f(x)|^{2} d x, \quad D[a]=H_{0}^{1}(U),
$$

so that Condition 2.1 is satisfied. We note that $H_{D}$ is the Schrödinger operator $H_{D}=$ $-\Delta+V$ with the Dirichlet boundary conditions.

Corollary 3.5. Suppose that $\Xi, \Gamma$, and $V$ satisfy the conditions of Theorem $[3.3, k \geq 2$. Let $U$ be a bounded domain with Lipschitz boundary $\partial U$, and let $\sigma$ be a real-valued function on $\partial U$ such that

$$
\sigma \in L_{q}(\partial U), \quad q>1 \text { for } k=2, \quad q=k-1 \text { for } k \geq 3 .
$$

Consider the quadratic form

$$
\begin{aligned}
h_{\sigma}[u]= & \int_{\Xi}\left(|\nabla u(x, y)|^{2}+V(x, y)|u(x, y)|^{2}\right) d x d y \\
& +\int_{\partial \Xi} \sigma(x)|u(x, y)|^{2} d S(x) d y, \quad D\left[h_{\sigma}\right]=H^{1}(\Xi),
\end{aligned}
$$

where $d S$ is an element of the surface area of $\partial U$. Then the spectrum of the Schrödinger operator $H_{\sigma}$ corresponding to the form $h_{\sigma}$ is absolutely continuous.

Indeed, in this case,

$$
a[f]=\int_{U}|\nabla f(x)|^{2} d x+\int_{\partial U} \sigma(x)|f(x)|^{2} d S(x), \quad D[a]=H^{1}(U) .
$$

The compactness of the embedding

$$
H^{1}(U) \subset L_{s}(\partial U), \quad \text { where } \quad s=\frac{2 k-2}{k-2} \text { for } k \geq 3, \quad s<\infty \text { for } k=2,
$$

and condition (3.4) imply an inequality

$$
\int_{\partial U}|\sigma(x)||f(x)|^{2} d S(x) \leq \varepsilon \int_{U}|\nabla f(x)|^{2} d x+c(\varepsilon) \int_{U}|f(x)|^{2} d x, \quad f \in H^{1}(U)
$$

(see, e.g., [10]). Therefore, the quadratic form $a$ is well defined. Finally, Condition 2.1 follows from the fact that the boundary $\partial U$ is Lipschitz (see Example 2.2). Observe that $H_{\sigma}$ is the Schrödinger operator $H_{\sigma}=-\Delta+V$ with the third type boundary condition (1.3). For $\sigma=0$ we get the Neumann boundary condition. 


\section{$\S 4$. Proof of the MAin Result}

4.1. The Thomas method. In this section we briefly describe the Thomas method (see [13]) of proving the absolute continuity of the spectrum (a more detailed exposition can be found in [2] or [6]). We assume that the conditions of Theorem 3.3 are fulfilled.

Consider the transformation

$$
(F u)(\xi, x, y)=|\widetilde{\Omega}|^{-1 / 2} \sum_{l \in \Gamma} e^{-i\langle\xi, y+l\rangle} u(x, y+l),
$$

known as the Floquet-Bloch or Gelfand transform. Here $\widetilde{\Omega}$ is an elementary cell of the dual lattice

$$
\widetilde{\Gamma}=\left\{n=\sum_{j=1}^{m} n_{j} \widetilde{b}_{j}, n_{j} \in \mathbb{Z}\right\}, \quad\left\langle b_{k}, \widetilde{b}_{j}\right\rangle=2 \pi \delta_{k j} .
$$

Equation (4.1) determines the transformation on a dense subset of smooth functions fast-decaying with respect to $y$. It can be extended to a unitary operator

$$
F: L_{2}(\Xi) \rightarrow \int_{\widetilde{\Omega}}^{\oplus} L_{2}(U \times \Omega) d \xi
$$

Moreover, $F$ maps $D\left[h_{0}\right]$ into $\int_{\widetilde{\Omega}}^{\oplus} X d \xi$, where

$$
X=\widetilde{H}^{1}\left(\Omega ; L_{2}(U)\right) \cap L_{2}(\Omega ; D[a])
$$

(we recall that the tilde over $H^{1}$ signalizes that the elements of this space satisfy the periodic boundary condition with respect to $y$ ). The operator $H$ can be expanded in a direct integral:

$$
F H F^{-1}=\int_{\widetilde{\Omega}}^{\oplus} H(\xi) d \xi .
$$

The operator $H(\xi)$ in the Hilbert space $L_{2}(U \times \Omega)=L_{2}\left(\Omega ; L_{2}(U)\right)$ is determined by its quadratic form

$$
\begin{aligned}
h(\xi)[v]=\int_{\Omega}\left(\left(\left(\nabla_{y}+i \xi\right) v(\cdot, y),\left(\nabla_{y}\right.\right.\right. & \left.+i \bar{\xi}) v(\cdot, y))_{L_{2}(U)}+a[v(\cdot, y)]\right) d y \\
& +\int_{U \times \Omega} V(x, y)|v(x, y)|^{2} d x d y, \quad D[h(\xi)]=X .
\end{aligned}
$$

Sometimes we shall use the operator-valued function $H(\xi)$ for complex $\xi$. It is easily seen that $H(\xi)^{*}=H(\xi)$ for real $\xi$.

The parameter $\xi$ is multivariate; it is convenient to select a special one-variate parameter. Let $b_{1}$ be the first vector of the basis of the lattice $\Gamma$. Since the formulation of Theorem 3.3 is invariant with respect to the dilatations of $y$, we can assume that $\left|b_{1}\right|=1$. Any vector $\xi \in \mathbb{R}^{m}$ can uniquely be expressed in the form $\xi=\xi_{1} b_{1}+\xi^{\prime}$, where $\left\langle\xi^{\prime}, b_{1}\right\rangle=0$. First, consider the integrals

$$
\int_{(0,2 \pi)}^{\oplus} H\left(\xi_{1} b_{1}+\xi^{\prime}\right) d \xi_{1} .
$$

Fix some $\xi^{\prime} \in \mathbb{R}^{m}$. The analytic Fredholm alternative guarantees that the spectrum of the operator (4.5) will not be absolutely continuous only if there exists a $\lambda$ that is an eigenvalue of $H\left(\xi_{1} b_{1}+\xi^{\prime}\right)$ for all $\xi_{1} \in \mathbb{C}$. Next, (4.4) implies that if the spectra of the operators (4.5) are absolutely continuous for all $\xi^{\prime}$, then the same is true for $H$. So, to prove Theorem 3.3 it suffices to show that for all $\lambda \in \mathbb{R}$ and all $\xi^{\prime} \in \mathbb{R}^{m}$ with $\xi^{\prime} \perp b_{1}$, the operator $\left(H\left(\xi_{1} b_{1}+\xi^{\prime}\right)-\lambda I\right)$ is invertible for some $\xi_{1} \in \mathbb{C}$. 
Theorem 4.1. Under the conditions of Theorem 3.3 , let $\left|b_{1}\right|=1$ and $\lambda \in \mathbb{R}$. Then for any $\xi^{\prime} \in \mathbb{R}^{m}$ with $\left\langle\xi^{\prime}, b_{1}\right\rangle=0$, there exists $\tau_{0}$ such that if $\pm \tau>\tau_{0}$, then the operator $\left(H\left((\pi+i \tau) b_{1}+\xi^{\prime}\right)-\lambda I\right)$ is invertible and

$$
\left\|\left(H\left((\pi+i \tau) b_{1}+\xi^{\prime}\right)-\lambda I\right)^{-1}\right\| \leq C|\tau|^{-1} .
$$

Remark 4.2. For simplicity, in what follows, we use rather loose notation $H(\tau)$ instead of $H\left((\pi+i \tau) b_{1}+\xi^{\prime}\right)$.

Remark 4.3. The conditions of Theorem 3.3 will not change if we add a constant to the potential. Thus, from now on we assume that $\lambda=0$.

Theorem 4.1 implies Theorem 3.3 . In the next section we shall prove Theorem 4.1, assuming some estimates of the quadratic form of the free operator $H_{0}(\tau)$ (Lemma 4.4 and Theorem 4.5). The proofs of Lemma 4.4 and Theorem 4.5 will be given in $\S \S 5$ and 6 , respectively.

4.2. Estimates. Let $H_{0}(\tau)$ be the operator $H(\tau)$ for $V=0$, and let $\left|H_{0}(\tau)\right|=$ $\left(H_{0}(\tau)^{*} H_{0}(\tau)\right)^{1 / 2}$. We denote the corresponding quadratic forms by $h_{0}(\tau)$ and $\left|h_{0}(\tau)\right|$; they all are defined on $\operatorname{Dom} h_{0}(\tau)=\operatorname{Dom}\left|h_{0}(\tau)\right|=X$ (see (4.3)).

Lemma 4.4. We have

$$
\left|h_{0}(\tau)\right|[u] \geq 2 \pi|\tau|\|u\|_{L_{2}(U \times \Omega)}^{2}, \quad u \in X .
$$

Theorem 4.5. Let $|\tau| \geq 1$. Then

$$
\left|h_{0}(\tau)\right|[u] \geq C\|u\|_{\frac{2 d-2}{d-2}(U \times \Omega)}^{2}, \quad u \in X,
$$

where the constant $C$ is independent of $\tau$ and $u$.

Combining Theorem 4.5 with Lemma 3.1 for $p=d-1$, we get the following statement.

Corollary 4.6. Let $V \in L_{d-1}(U \times \Omega)$. Then for any $\varepsilon>0$, there exists a constant $c(\varepsilon)$ such that

$$
\begin{array}{r}
\int_{U \times \Omega}|V u \bar{v}| d x d y \leq \varepsilon\left(\left|h_{0}(\tau)\right|[u]\right)^{1 / 2}\left(\left|h_{0}(\tau)\right|[v]\right)^{1 / 2}+c(\varepsilon)\|u\|_{L_{2}(U \times \Omega)}\|v\|_{L_{2}(U \times \Omega)}, \\
u, v \in X .
\end{array}
$$

Proof of Theorem 4.1. Let $u \in \operatorname{Dom} H(\tau) \subset X$. We are going to prove that $\|H(\tau) u\|_{L_{2}} \geq$ $C|\tau|\|u\|_{L_{2}},|\tau|>\tau_{0}$. Put

$$
v=\left(H_{0}(\tau)^{*}\right)^{-1}\left|H_{0}(\tau)\right| u=\left|H_{0}(\tau)\right|^{-1} H_{0}(\tau) u \in X .
$$

Observe that the operators $H_{0}(\tau), H_{0}(\tau)^{*}$, and $\left|H_{0}(\tau)\right|$ commute, because in the discrete Fourier representation they become the operators of multiplication by their symbols. So, $\|v\|_{L_{2}(U \times \Omega)}=\|u\|_{L_{2}(U \times \Omega)}$ and

$$
\left|h_{0}(\tau)\right|[v]=h_{0}(\tau)[u, v]=\left|h_{0}(\tau)\right|[u] .
$$

Using Corollary 4.6 with $\varepsilon=1 / 2$, we get

$$
\int_{U \times \Omega}|V u \bar{v}| d x d y \leq \frac{1}{2}\left|h_{0}(\tau)\right|[u]+C\|u\|_{L_{2}(U \times \Omega)}^{2} .
$$

Hence,

$$
\begin{aligned}
|(H(\tau) u, v)| & =\left|h_{0}(\tau)[u, v]+\int_{U \times \Omega} V u \bar{v} d x d y\right|=|| h_{0}(\tau)\left|[u]+\int_{U \times \Omega} V u \bar{v} d x d y\right| \\
& \geq \frac{1}{2}\left|h_{0}(\tau)\right|[u]-C\|u\|_{L_{2}(U \times \Omega)}^{2} \geq(\pi|\tau|-C)\|u\|_{L_{2}(U \times \Omega)}^{2},
\end{aligned}
$$


where we have used Lemma 4.4 at the last step. On the other hand,

$$
|(H(\tau) u, v)| \leq\|H(\tau) u\|_{L_{2}(U \times \Omega)}\|v\|_{L_{2}(U \times \Omega)},
$$

which implies that

$$
\|H(\tau) u\|_{L_{2}(U \times \Omega)} \geq(\pi|\tau|-C)\|u\|_{L_{2}(U \times \Omega)}
$$

for all $u \in \operatorname{Dom} H(\tau)$.

We note that if $V \in L_{\infty}$, then Lemma 4.4 (without Theorem 4.5) immediately yields Theorem 4.1 (see also 4, Theorem 5.4.9]). Indeed, if $V$ is bounded, then

$|(H(\tau) u, v)| \geq\left|h_{0}(\tau)\right|[u]-\|V\|_{L_{\infty}(U \times \Omega)}\|u\|_{L_{2}(U \times \Omega)}^{2} \geq\left(2 \pi|\tau|-\|V\|_{L_{\infty}(U \times \Omega)}\right)\|u\|_{L_{2}(U \times \Omega)}^{2}$,

which gives $\left\|H(\tau)^{-1}\right\| \leq\left(2 \pi|\tau|-\|V\|_{L_{\infty}}\right)^{-1}$. As we shall see below, the proof of Lemma 4.4 is quite easy. However, to take the potentials $V \in L_{d-1 \text {,loc }}$ into consideration, we need sharper lower estimates of $\left|h_{0}(\tau)\right|$ (Theorem 4.5). The proof of Theorem 4.5) is rather technical.

We also remark that, for $d \geq 4$, the proof of Theorem 4.1 (and, hence, of Theorem 3.3) can be simplified; see Subsection 7.2 below.

\section{§5. Symbol of a FREe OPERATOR}

The free operator $H_{0}(\tau)$ corresponds to the quadratic form

$$
h_{0}(\tau)[u]=\int_{\Omega}\left(\left(\left(\nabla_{y}+i \xi\right) u(\cdot, y),\left(\nabla_{y}+i \bar{\xi}\right) u(\cdot, y)\right)_{L_{2}(U)}+a[u(\cdot, y)]\right) d y, \quad u \in X,
$$

where, we recall, $\xi=(\pi+i \tau) b_{1}+\xi^{\prime}$. Let $\left\{\psi_{j}\right\}_{j=1}^{\infty}$ be an orthonormal basis in $L_{2}(U)$ consisting of eigenfunctions of the operator $\mathcal{A}$ that corresponds to the quadratic form $a$,

$$
\mathcal{A} \psi_{j}=\mu_{j} \psi_{j}, \quad \mu_{1} \leq \mu_{2} \leq \cdots \text { are the eigenvalues. }
$$

Then the functions

$$
\left\{|\Omega|^{-1 / 2} \psi_{j}(x) e^{i\langle n, y\rangle}\right\}_{j \in \mathbb{N}, n \in \widetilde{\Gamma}}
$$

form an orthonormal basis in $L_{2}(U \times \Omega)$. The functions (5.1) are eigenfunctions of $H_{0}(\tau)$. The corresponding eigenvalues look like this:

$$
h_{j, n}(\tau)=\langle n+\xi, n+\bar{\xi}\rangle+\mu_{j}=\left|n+\pi b_{1}+\xi^{\prime}\right|^{2}+\mu_{j}-\tau^{2}+2 i \tau\left\langle n+\pi b_{1}, b_{1}\right\rangle
$$

(we have used the assumptions $\left|b_{1}\right|=1$ and $\left\langle\xi^{\prime}, b_{1}\right\rangle=0$ ). Hence,

$$
\operatorname{Im} h_{j, n}(\tau)=2 \tau\left(n_{1}+\pi\right),
$$

where $n_{1}:=\left\langle n, b_{1}\right\rangle$. We note that $n_{1} \in 2 \pi \mathbb{Z}$ by the definition of the dual lattice (4.2). Thus,

$$
\left|\operatorname{Im} h_{j, n}(\tau)\right| \geq 2 \pi|\tau|
$$

and moreover,

$$
\left|\operatorname{Im} h_{j, n}(\tau)\right| \geq \frac{2}{3}|\tau|\left(\left|n_{1}\right|+1\right) .
$$

Proof of Lemma 4.4. The functions (5.1) are also eigenfunctions of $\left|H_{0}(\tau)\right|$; the corresponding eigenvalues are $\left|h_{j, n}(\tau)\right|$. So, (4.6) follows from (5.2). 
To prove Theorem 4.5, we need sharper estimates of the symbol of $H_{0}(\tau)$. For every $\tau$, consider the following three sets of pairs of indices $(j, n) \in \mathbb{N} \times \widetilde{\Gamma}$ :

$$
\begin{aligned}
& J_{1}=\left\{(j, n):\left|n+\pi b_{1}+\xi^{\prime}\right|^{2}+\mu_{j} \leq \frac{1}{2}\left(n^{2}+\mu_{j}+\gamma\right)\right\}, \\
& J_{2}=\left\{(j, n): n^{2}+\mu_{j}+\gamma \leq 4 \tau^{2}\right\}, \\
& J_{3}=\left\{(j, n):\left|n+\pi b_{1}+\xi^{\prime}\right|^{2}+\mu_{j}>\frac{1}{2}\left(n^{2}+\mu_{j}+\gamma\right)>2 \tau^{2}\right\} .
\end{aligned}
$$

Obviously,

$$
\mathbb{N} \times \widetilde{\Gamma}=J_{1} \cup J_{2} \cup J_{3} .
$$

Lemma 5.1. a) The set $J_{1}$ is finite and does not depend on $\tau$.

b) If $(j, n) \in J_{2}$, then

$$
\left|\tau h_{j, n}(\tau)\right| \geq \frac{1}{6}\left(\left|n_{1}\right|+1\right)\left(n^{2}+\mu_{j}+\gamma\right)
$$

c) If $(j, n) \in J_{3}$, then

$$
\left|h_{j, n}(\tau)\right|>\frac{1}{4}\left(n^{2}+\mu_{j}+\gamma\right) .
$$

Proof. Statement a) is obvious.

Statement b) follows from (5.3) and the condition $4 \tau^{2} \geq n^{2}+\mu_{j}+\gamma$.

c) Let $(j, n) \in J_{3}$. Then

$$
\operatorname{Re} h_{j, n}(\tau)=\left|n+\pi b_{1}+\xi^{\prime}\right|^{2}+\mu_{j}-\tau^{2}>\frac{1}{4}\left(n^{2}+\mu_{j}+\gamma\right) .
$$

\section{§6. Proof of Theorem 4.5}

6.1. The embedding $X \subset L_{\frac{2 d-2}{d-2}}$. Let $u \in X$. The function $u$ can be represented as a linear combination of the basis functions (5.1):

$$
u(x, y)=|\Omega|^{-1 / 2} \sum_{j \in \mathbb{N}, n \in \widetilde{\Gamma}} c_{j, n} \psi_{j}(x) e^{i\langle n, y\rangle} .
$$

Then

$$
\left|h_{0}(\tau)\right|[u]=\sum_{j, n}\left|h_{j, n}(\tau)\right|\left|c_{j, n}\right|^{2} .
$$

Lemma 6.1. Let $u \in X$, and let

$$
u(x, y)=|\Omega|^{-1 / 2} \sum_{(j, n) \in J_{2}} c_{j, n} \psi_{j}(x) e^{i\langle n, y\rangle}
$$

(we emphasize that the summation is only over $J_{2}$ ). Then

$$
\|u\|_{\frac{2 d-2}{d-2}(U \times \Omega)}^{2} \leq C\left|h_{0}(\tau)\right|[u] .
$$

This lemma will be proved in the next section.

Lemma 6.2. Let $u \in X$, and let

$$
u(x, y)=|\Omega|^{-1 / 2} \sum_{(j, n) \in J_{3}} c_{j, n} \psi_{j}(x) e^{i\langle n, y\rangle}
$$

(the summation is only over $J_{3}$ ). Then

$$
\|u\|_{\frac{2 d-2}{d-2}(U \times \Omega)}^{2} \leq C\left|h_{0}(\tau)\right|[u] .
$$


Proof. By Corollary 2.6, we have

$$
\begin{aligned}
\|u\|_{L_{\frac{2 d}{d-2}}^{2}(U \times \Omega)}^{2} & \leq C \int_{\Omega}\left(\left\|\nabla_{y} u(\cdot, y)\right\|_{L_{2}(U)}^{2}+a[u(\cdot, y)]+\gamma\|u(\cdot, y)\|_{L_{2}(U)}^{2}\right) d y \\
& =C \sum_{(j, n) \in J_{3}}\left(n^{2}+\mu_{j}+\gamma\right)\left|c_{j, n}\right|^{2} .
\end{aligned}
$$

Using (6.2) and Lemma $5.1 \mathrm{c}$ ), we see that $\|u\|_{L_{\frac{2 d}{d-2}}^{2}(U \times \Omega)}^{2} \leq 4 C\left|h_{0}(\tau)\right|[u]$, which yields the statement of the lemma, because the set $U \times \Omega$ is bounded.

Proof of Theorem 4.5. The function $u$ can be expressed in the form $u=u_{1}+u_{2}+u_{3}$, where

$$
u_{k}(x, y)=|\Omega|^{-1 / 2} \sum_{(j, n) \in J_{k}} c_{j, n} \psi_{j}(x) e^{i\langle n, y\rangle}, \quad k=1,2,3 .
$$

This is possible because $J_{1} \cup J_{2} \cup J_{3}=\mathbb{N} \times \widetilde{\Gamma}$. Also, we can assume that for every pair $(j, n)$, the corresponding coefficient $c_{j, n}$ is nonzero for at most one of the functions $u_{1}$, $u_{2}$, or $u_{3}$.

The set $J_{1}$ is finite and does not depend on $\tau$. Since the corresponding linear subspace is finite-dimensional, the $L_{2}$-norm in this space is equivalent to the $L_{\frac{2 d-2}{d-2}}$-norm. Using (4.6), for $|\tau| \geq 1$ we get

$$
\left\|u_{1}\right\|_{L_{\frac{2 d-2}{d-2}}(U \times \Omega)} \leq C\left(\left|h_{0}(\tau)\right|\left[u_{1}\right]\right)^{1 / 2},
$$

where $C$ does not depend on $\tau$. For $u_{2}$ we have Lemma6.1, and for $u_{3}$ we use Lemma 6.2. Finally, we have the trivial inequalities

$$
\|u\|_{L_{\frac{2 d-2}{d-2}}(U \times \Omega)} \leq\left\|u_{1}\right\|_{L_{\frac{2 d-2}{d-2}}(U \times \Omega)}+\left\|u_{2}\right\|_{L_{\frac{2 d-2}{d-2}}(U \times \Omega)}+\left\|u_{3}\right\|_{L_{\frac{2 d-2}{d-2}}(U \times \Omega)},
$$

and $\left|h_{0}(\tau)\right|\left[u_{k}\right] \leq\left|h_{0}(\tau)\right|[u], k=1,2,3$.

It only remains to prove Lemma 6.1

6.2. Proof of Lemma 6.1, We introduce the notation

$$
\Omega^{\prime}=\left\{y^{\prime}=\sum_{j=2}^{m} y_{j} b_{j}, y_{j} \in(0,1)\right\} \subset \mathbb{R}^{m-1}, \quad G=\mathbb{R}^{k} \times \Omega^{\prime}, \quad m \geq 2 ;
$$

$G=\mathbb{R}^{k}, m=1$. For all $m$ we have $G \subset \mathbb{R}^{d-1}$ and $\mathbb{R}^{k} \times \Omega=G \times(0,1)$.

Next, by $H^{1 / 2}((0,1) ; Y)$, where $Y$ is a Hilbert space, we mean the space of functions of the form

$$
f\left(y_{1}\right)=\sum_{n_{1} \in 2 \pi \mathbb{Z}} f_{n_{1}} e^{i n_{1} y_{1}},
$$

where $y_{1} \in(0,1), f_{n_{1}} \in Y$, with the norm

$$
\|f\|_{H^{1 / 2}((0,1) ; Y)}^{2}=\sum_{n_{1} \in 2 \pi \mathbb{Z}}\left(\left|n_{1}\right|+1\right)\left\|f_{n_{1}}\right\|_{Y}^{2} .
$$

It is well known that

$$
H^{1 / 2}((0,1) ; Y) \subset L_{s}((0,1) ; Y), \quad s<\infty .
$$

Lemma 6.3. Let $\Pi$ be the extension operator introduced in Subsection 2.2. Then

$$
\|\Pi u\|_{H^{1 / 2}\left((0,1) ; L_{2}(G)\right)}^{2} \leq C|\tau|^{-1}\left|h_{0}(\tau)\right|[u], \quad u \in X .
$$


Proof. We expand $u$ in the series (6.1). If $m \geq 2$, then, using the orthogonality of the exponentials $e^{i\left\langle n^{\prime}, y^{\prime}\right\rangle}$ in $L_{2}\left(\Omega^{\prime}\right)$, we obtain

$$
\begin{aligned}
\|\Pi u\|_{H^{1 / 2}\left((0,1) ; L_{2}(G)\right)}^{2} & =|\Omega|^{-1} \sum_{n_{1}}\left(\left|n_{1}\right|+1\right) \int_{\mathbb{R}^{k}} \int_{\Omega^{\prime}}\left|\sum_{j, n^{\prime}} c_{j, n} \Pi_{0} \psi_{j}(x) e^{i\left\langle n^{\prime}, y^{\prime}\right\rangle}\right|^{2} d y^{\prime} d x \\
& =\left|\Omega^{\prime}\right||\Omega|^{-1} \sum_{n}\left(\left|n_{1}\right|+1\right) \int_{\mathbb{R}^{k}}\left|\Pi_{0} \sum_{j} c_{j, n} \psi_{j}(x)\right|^{2} d x .
\end{aligned}
$$

If $m=1$, then, by definition (6.5),

$$
\|\Pi u\|_{H^{1 / 2}\left((0,1) ; L_{2}\left(\mathbb{R}^{k}\right)\right)}^{2}=\sum_{n}(|n|+1)\left\|\Pi_{0} \sum_{j} c_{j, n} \psi_{j}\right\|_{L_{2}\left(\mathbb{R}^{k}\right)}^{2} .
$$

The statement of the lemma now follows from the inequality

$$
\left\|\Pi_{0} \sum_{j} c_{j, n} \psi_{j}\right\|_{L_{2}\left(\mathbb{R}^{k}\right)}^{2} \leq\left\|\Pi_{0}\right\|_{L_{2}(U) \rightarrow L_{2}\left(\mathbb{R}^{k}\right)}^{2} \sum_{j}\left|c_{j, n}\right|^{2}
$$

and from (6.2) and (5.3).

Lemma 6.4. Under the conditions of Lemma 6.1, we have

$$
\|\Pi u\|_{H^{1 / 2}\left((0,1) ; H^{1}(G)\right)}^{2} \leq C|\tau|\left|h_{0}(\tau)\right|[u] .
$$

Proof. By analogy with the preceding lemma, we have

$$
\begin{array}{r}
\|\Pi u\|_{H^{1 / 2}\left((0,1) ; H^{1}(G)\right)}^{2}=|\Omega|^{-1} \sum_{n_{1}}\left(\left|n_{1}\right|+1\right) \int_{\mathbb{R}^{k}} \int_{\Omega^{\prime}}\left(\left|\sum_{j, n^{\prime}} c_{j, n} \Pi_{0} \psi_{j}(x) e^{i\left\langle n^{\prime}, y^{\prime}\right\rangle}\right|^{2}\right. \\
\left.+\left|\nabla_{x} \sum_{j, n^{\prime}} c_{j, n} \Pi_{0} \psi_{j}(x) e^{i\left\langle n^{\prime}, y^{\prime}\right\rangle}\right|^{2}+\left|\nabla_{y^{\prime}} \sum_{j, n^{\prime}} c_{j, n} \Pi_{0} \psi_{j}(x) e^{i\left\langle n^{\prime}, y^{\prime}\right\rangle}\right|^{2}\right) d y^{\prime} d x \\
=\frac{\left|\Omega^{\prime}\right|}{|\Omega|} \sum_{n}\left(\left|n_{1}\right|+1\right)\left(\left\|\Pi_{0} \sum_{j} c_{j, n} \psi_{j}\right\|_{H^{1}\left(\mathbb{R}^{k}\right)}^{2}+\left|n^{\prime}\right|^{2}\left\|\Pi_{0} \sum_{j} c_{j, n} \psi_{j}\right\|_{L_{2}\left(\mathbb{R}^{k}\right)}^{2}\right),
\end{array}
$$

where we assume that the summation is only over the pairs $(j, n) \in J_{2}$; for $m=1$ the last term on the right-hand side vanishes. Condition 2.1 gives

$$
\left\|\Pi_{0} \sum_{j} c_{j, n} \psi_{j}\right\|_{H^{1}\left(\mathbb{R}^{k}\right)}^{2} \leq C_{0} \sum_{j}\left(\mu_{j}+\gamma\right)\left|c_{j, n}\right|^{2} .
$$

Taking (6.7) into account, we get

$$
\|\Pi u\|_{H^{1 / 2}\left((0,1) ; H^{1}(G)\right)}^{2} \leq C_{1}\left|\Omega^{\prime}\right||\Omega|^{-1} \sum_{(j, n) \in J_{2}}\left(\left|n_{1}\right|+1\right)\left(\left|n^{\prime}\right|^{2}+\mu_{j}+\gamma\right)\left|c_{j, n}\right|^{2},
$$

where $C_{1}=\max \left(C_{0},\left\|\Pi_{0}\right\|_{L_{2} \rightarrow L_{2}}^{2}\right)$. Now we refer to Lemma $\left.5.1 \mathrm{~b}\right)$ and to (6.2).

Lemma 6.5. a) $\left\|f^{2}\right\|_{W_{1}^{1}(G)} \leq 3\|f\|_{H^{1}(G)}\|f\|_{L_{2}(G)}, f \in H^{1}(G)$.

b) Let $q \geq 2$. Then

$$
\|u\|_{L_{q}\left((0,1) ; L_{\frac{2 d-2}{d-2}}(G)\right)}^{2} \leq C\|u\|_{L_{q}\left((0,1) ; H^{1}(G)\right)}\|u\|_{L_{q}\left((0,1) ; L_{2}(G)\right)}, \quad u \in L_{q}\left((0,1) ; H^{1}(G)\right) .
$$


Proof. a) We have

$$
\begin{aligned}
\left\|f^{2}\right\|_{W_{1}^{1}(G)} & =\int_{G}\left(\left|\nabla\left(f^{2}\right)\right|+\left|f^{2}\right|\right) d x d y^{\prime} \leq \int_{G}|f|(2|\nabla f|+|f|) d x d y^{\prime} \\
& \leq\left(2\|\nabla f\|_{L_{2}(G)}+\|f\|_{L_{2}(G)}\right)\|f\|_{L_{2}(G)} \leq 3\|f\|_{H^{1}(G)}\|f\|_{L_{2}(G)} .
\end{aligned}
$$

b) Observe that

$$
\|u\|_{L_{q}\left((0,1) ; L_{\frac{2 d-2}{d-2}}^{2}(G)\right)}^{2}=\left\|u^{2}\right\|_{L_{q / 2}\left((0,1) ; L_{\frac{d-1}{d-2}}(G)\right)} .
$$

The embedding theorem $W_{1}^{1}(G) \subset L_{\frac{d-1}{d-2}}(G)$ gives the estimate

$$
\left\|u^{2}\right\|_{L_{q / 2}\left((0,1) ; L_{\frac{d-1}{d-2}}(G)\right)} \leq C\left\|u^{2}\right\|_{L_{q / 2}\left((0,1) ; W_{1}^{1}(G)\right)} .
$$

Next, by a),

$$
\begin{aligned}
\left\|u^{2}\right\|_{L_{q / 2}\left((0,1) ; W_{1}^{1}(G)\right)}^{q} & =\left(\int_{0}^{1}\left\|u^{2}\left(y_{1}\right)\right\|_{W_{1}^{1}(G)}^{q / 2} d y_{1}\right)^{2} \\
& \leq 3^{q}\left(\int_{0}^{1}\left\|u\left(y_{1}\right)\right\|_{H^{1}(G)}^{q / 2}\left\|u\left(y_{1}\right)\right\|_{L_{2}(G)}^{q / 2} d y_{1}\right)^{2} \\
& \leq 3^{q} \int_{0}^{1}\left\|u\left(y_{1}\right)\right\|_{H^{1}(G)}^{q} d y_{1} \int_{0}^{1}\left\|u\left(y_{1}\right)\right\|_{L_{2}(G)}^{q} d y_{1} \\
& =3^{q}\|u\|_{L_{q}\left((0,1) ; H^{1}(G)\right)}^{q}\|u\|_{L_{q}\left((0,1) ; L_{2}(G)\right)}^{q} .
\end{aligned}
$$

Remark 6.6. The proof of Lemma6.5 is similar to the proof of Ladyzhenskaya's inequality $\|u\|_{L_{4}}^{2} \leq c\|u\|_{H^{1}}\|u\|_{L_{2}}$ in a two-dimensional domain; see [5].

Proof of Lemma 6.1. By Lemma 6.5 b) with $q=(2 d-2) /(d-2)$, we have

$$
\begin{aligned}
\|u\|_{L_{\frac{2 d-2}{d-2}}(U \times \Omega)}^{2} & \leq\|\Pi u\|_{L_{\frac{2 d-2}{d-2}}(G \times(0,1))}^{2}=\|\Pi u\|_{L_{\frac{2 d-2}{d-2}}\left((0,1) ; L_{\frac{2 d-2}{d-2}}(G)\right)}^{2} \\
& \leq C\|\Pi u\|_{L_{\frac{2 d-2}{d-2}}\left((0,1) ; H^{1}(G)\right)}\|\Pi u\|_{\frac{2 d-2}{d-2}}\left((0,1) ; L_{2}(G)\right) .
\end{aligned}
$$

Next, using (6.6) with $s=(2 d-2) /(d-2)$ and Lemma 6.3, we obtain

$$
\|\Pi u\|_{L_{\frac{2 d-2}{d-2}}^{2}\left((0,1) ; L_{2}(G)\right)}^{2} \leq C\|\Pi u\|_{H^{1 / 2}\left((0,1) ; L_{2}(G)\right)}^{2} \leq C^{\prime}|\tau|^{-1}\left|h_{0}(\tau)\right|[u],
$$

and, finally, (6.6) and Lemma 6.4 yield

$$
\|\Pi u\|_{L_{\frac{2 d-2}{d-2}}^{2}\left((0,1) ; H^{1}(G)\right)}^{2} \leq C\|\Pi u\|_{H^{1 / 2}\left((0,1) ; H^{1}(G)\right)}^{2} \leq C^{\prime}\left|\tau \| h_{0}(\tau)\right|[u] .
$$

Multiplying the last two inequalities and using (6.8), we obtain (6.3).

\section{$\S 7$. Comments}

7.1. Assume that

$$
V \in L_{r}\left((0,1) ; L_{d-1}\left(U \times \Omega^{\prime}\right)\right), \quad r>1,
$$

where $\Omega^{\prime}$ is the set introduced in (6.4). By using the embedding theorem,

$$
H^{1}\left(\mathbb{R}^{k} \times \Omega\right) \subset L_{s}\left((0,1) ; L_{\frac{2 d-2}{d-2}}(G)\right), \quad s<\infty
$$

(see [1, §10]), it can be shown that the form (3.3) is closed and semibounded, and hence, the operator $H=H_{0}+V$ is well defined. It can be seen that for such potentials, Theorem 3.3 can be proved as above. 
7.2. For $d=2$ (the two-dimensional plane or a strip region) our method gives absolute continuity for $V \in L_{r, \text { loc }}, r>1$. As was mentioned before, this result is well known.

For $d \geq 4$ the proof of the main Theorem 3.3 can be simplified. By analogy with Lemma [5.1, it can be shown that for all $j, n$, we have

$$
\left|h_{j, n}(\tau)\right| \geq c \sqrt{n^{2}+\mu_{j}+\gamma}\left(\left|n_{1}\right|+1\right), \quad|\tau|>\tau_{0} .
$$

Therefore,

$$
\|\Pi u\|_{H^{1}\left((0,1), H^{1}(G)\right)} \leq C\left\|H_{0}(\tau) u\right\|_{L_{2}(U \times \Omega)}, \quad u \in \operatorname{Dom} H_{0}(\tau), \quad|\tau|>\tau_{0} .
$$

The embeddings $H^{1}(G) \subset L_{\frac{2 d-2}{d-3}}(G)$ and $H^{1}(0,1) \subset C[0,1] \subset L_{\frac{2 d-2}{d-3}}(0,1)$ give us the estimate

$$
\|u\|_{L_{\frac{2 d-2}{d-3}}(U \times \Omega)} \leq C\left\|H_{0}(\tau) u\right\|_{L_{2}(U \times \Omega)}, \quad|\tau|>\tau_{0} .
$$

As in Lemma 3.1, formula (7.1) yields

$$
\|V u\|_{L_{2}(U \times \Omega)} \leq \varepsilon\left\|H_{0}(\tau) u\right\|_{L_{2}(U \times \Omega)}+c(\varepsilon)\|u\|_{L_{2}(U \times \Omega)}, \quad|\tau|>\tau_{0},
$$

which, in its turn, implies (4.7). However, for $d=3$ these considerations allow us to prove the absolute continuity of $H$ only if $V \in L_{p}(U \times \Omega), p>2$.

7.3. For the case of a Schrödinger operator with the third type boundary condition, it is also of interest to consider the case of $\sigma$ depending on $y$. Let

$$
\begin{gathered}
h_{\sigma}[u]=\int_{\Xi}\left(|\nabla u(x, y)|^{2}+V(x, y)|u(x, y)|^{2}\right) d x d y+\int_{\partial \Xi} \sigma(x, y)|u(x, y)|^{2} d S(x) d y, \\
D\left[h_{\sigma}\right]=H^{1}(\Xi), \quad \sigma(x, y+l)=\sigma(x, y) \quad \text { for all } \quad l \in \Gamma .
\end{gathered}
$$

It is known that the spectrum of the corresponding operator $H_{\sigma}$ is absolutely continuous if $k=1$ (when $V$ and $\sigma$ belong to the corresponding $L_{p^{-}}$-spaces, see [9]). A similar problem was treated in [10]: absolute continuity was proved in the case of a potential concentrated on a periodic system of hypersurfaces. However, the methods of $[9]$ and [10] are not applicable to our case.

We also note that the operator $H_{\sigma}$ is absolutely continuous whenever it is "even". Assume for simplicity that the lattice of periods is cubic, $\Gamma=\mathbb{Z}^{m}$. If $V$ and $\sigma$ are invariant with respect to the reflection along one of the coordinate axes,

$$
V\left(x, y_{1}, y^{\prime}\right)=V\left(x, 1-y_{1}, y^{\prime}\right), \quad \sigma\left(x, y_{1}, y^{\prime}\right)=\sigma\left(x, 1-y_{1}, y^{\prime}\right),
$$

then the spectrum of $H_{\sigma}$ is absolutely continuous; see [14, 12.

The question concerning the absolute continuity of $H_{\sigma}$ (without assuming the operator to be even) for $k \geq 2$ remains open (even for $V=0, \sigma \in C^{\infty}\left(\partial U \times \mathbb{R}^{m}\right)$ ).

7.4. By analogy with the proof of Theorem 3.3, the following result can be obtained.

Theorem 7.1. Let $M$ be a smooth compact Riemannian manifold without boundary, and let $\operatorname{dim} M=k, \Xi=M \times \mathbb{R}^{m}, \operatorname{dim} \Xi=d=k+m \geq 3$. Let $-\Delta$ be the Laplace-Beltrami operator on $\Xi$. Let $\Gamma$ be a lattice in $\mathbb{R}^{m}$. Suppose that the potential $V$ in $\Xi$ satisfies (1.2)

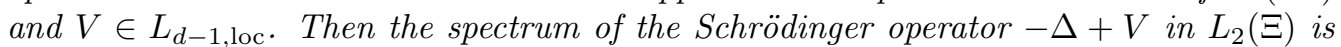
absolutely continuous.

If $M$ is a compact manifold with boundary, then a similar result is valid for the Schrödinger operator with suitable boundary conditions. 


\section{REFERENCES}

[1] O. V. Besov, V. P. Il'in, and S. M. Nikol'skiı̆, Integral representations of functions, and embedding theorems, 2nd ed., Nauka, Moscow, 1996; English transl. of 1st ed., V. H. Winston and Sons, Washington, DC; Halsted Press, New York, Vol. I, 1978; Vol. II, 1979. MR.1450401 (98b:46037) MR0519341 (80f:46030a) MR0521808 (80f:46030b)

[2] M. Sh. Birman and T. A. Suslina, Periodic magnetic Hamiltonian with variable metric. The problem of absolute continuity, Algebra i Analiz 11 (1999), no. 2, 1-40; English transl., St. Petersburg Math. J. 11 (2000), no. 2, 203-232. MR.1702587 (2000i:35026)

[3] I. Kiba, Absolute continuity of a periodic Schrödinger operator in waveguide with constant section, Bachelor's Paper, S.-Peterburg. Univ., Fiz. Fak., St. Petersburg, 2001. (Russian)

[4] P. Kuchment, Floquet theory for partial differential equations, Oper. Theory Adv. Appl., vol. 60, Birkhäuser, Basel, 1993. MR1232660 (94h:35002)

[5] O. A. Ladyzhenskaya, Mathematical problems in the dynamics of a viscous incompressible fluid, Fizmatgiz, Moscow, 1961; English transl., The mathematical theory of viscous incompressible flow, Gordon and Breach Sci. Publishers, New York-London, 1963. MR:0155092 (27:5034a); MR0155093 $(27: 5034 \mathrm{~b})$

[6] M. Reed and B. Simon, Methods of modern mathematical physics. IV. Analysis of operators, Acad. Press, New York-London, 1978. MR0493421 (58:12429c)

[7] E. Shargorodsky and A. V. Sobolev, Quasiconformal mappings and periodic spectral problems in dimension two, J. Anal. Math. 91 (2003), 67-103. MR2037402(2005b:35202)

[8] E. M. Stein, Singular integrals and differentiability properties of functions, Princeton Math. Ser., No. 30, Princeton Univ. Press, Princeton, NJ, 1970. MR0290095 (44:7280)

[9] T. A. Suslina, On the absence of eigenvalues of a periodic matrix Schrödinger operator in a layer, Russ. J. Math. Phys. 8 (2001), no. 4, 463-486. (English) MR1932011(2003k:81057)

[10] T. A. Suslina and R. G. Shterenberg, Absolute continuity of the spectrum of the Schrödinger operator with the potential concentrated on a periodic system of hypersurfaces, Algebra i Analiz 13 (2001), no. 5, 197-240; English transl., St. Petersburg Math. J. 13 (2002), no. 5, 859-891. MR1882869 (2002m:35172)

[11] Absolute continuity of the spectrum of the magnetic Schrödinger operator with metric in a two-dimensional periodic waveguide, Algebra i Analiz 14 (2002), no. 2, 159-206; English transl., St. Petersburg Math. J. 14 (2003), no. 2, 305-343. MR1925885 (2003h:35185)

[12] M. Tikhomirov and N. Filonov, Absolute continuity of the "even" periodic Schrödinger operator with nonsmooth coefficients, Algebra i Analiz 16 (2004), no. 3, 201-210; English transl., St. Petersburg Math. J. 16 (2005), no. 3, 583-589. MR2083570(2005f:35056)

[13] L. Thomas, Time dependent approach to scattering from impurities in a crystal, Comm. Math. Phys. 33 (1973), 335-343. MR0334766 (48:13084)

[14] L. Friedlander, Absolute continuity of the spectra of periodic waveguides, Waves in Periodic and Random Media (South Hadley, MA, 2002), Contemp. Math., vol. 339, Amer. Math. Soc., Providence, RI, 2003, pp. 37-42. MR 2042530 (2005a:35211)

[15] Z. Shen, On absolute continuity of the periodic Schrödinger operators, Internat. Math. Res. Notices 2001, no. 1, 1-31. MR1809495 (2002a:47078)

St. Petersburg Branch, Steklov Mathematical Institute, Russian Academy of Sciences, Fontanka 27, 191023 St. Petersburg, Russia

E-mail address: ilya.kachkovskiy@gmail.com

St. Petersburg Branch, Steklov Mathematical Institute, Russian Academy of Sciences, Fontanka 27, 191023 St. Petersburg, Russia

E-mail address: filonov@pdmi.ras.ru

Received 6/AUG/2008

Translated by THE AUTHORS 